\title{
Behandlung der Talusfraktur
}

\author{
Martinus Richter, Stefan Zech
}

\section{Zusammenfassung}

Talusfrakturen gehören zu den seltenen Fußfrakturen, führen aber potenziell zu schweren Komplikationen. Die gängigen Frakturklassifikationen beruhen auf konventionellen Röntgenaufnahmen, die Computertomografie ist jedoch bei jeder Fraktur obligat. Offene Frakturen, Luxationen oder Extrusionen müssen notfallmäßig reponiert und stabilisiert werden. Alle dislozierten Talusfrakturen stellen eine absolute Operationsindikation dar. Die Zugangswahl wird vom Frakturtyp und dem Weichteilschaden bestimmt. Die anatomische Reposition der Gelenkflächen und der Talusform und die Stabilisierung mit Zugschrauben stellt meistens die Methode der Wahl dar. Das Ergebnis wird vom Dislokationsgrad bzw. dem Weichteilschaden, aber oftmals auch von der unzulänglichen Versorgung beeinflusst. Verzögerte Konsolidierungen, Pseudarthrosen und sekundäre Fehlstellungen sollten möglichst frühzeitig Gelenk erhaltend korrigiert und notwendige Arthrodesen auf die betroffenen Gelenke beschränkt werden. Dieser Artikel behandelt detailliert Klassifikation, operative Behandlung, Indikationsstellung und Komplikationen von Talushalsfrakturen. Die Körper- und Kopffrakturen werden ebenfalls diskutiert.

\section{Einleitung}

Frakturen des Sprungbeines sind relativ seltene Frakturen. Sie werden mit einer

OP-JOURNAL 2007; 23: 104-109

(C) Georg Thieme Verlag KG Stuttgart • New York

\section{Treatment of Talar Fractures}

Fractures of the talus are a relatively uncommon fracture of the foot, but they have potentially serious complications. The classifications of fractures are based on conventional X-rays, but computed tomography is necessary for treatment decisions. Open fractures, displaced fracture dislocations, or extrusion of the talus must be reduced and stabilised as an emergency procedure. Operative treatment is indicated in all displaced central fractures. The use of standardised approaches depends on the type of fracture and the soft tissue lesions. Precise anatomic reduction of all facets and reconstruction of the shape of the talus and stabilisation with interfragmentary lag screws is the method of choice in almost all fractures. This procedure allows for early mobilisation postoperatively. The outcome is related to the degree of fracture displacement and the soft tissue lesions but may be poor due to inadequate treatment. Talus malunion, non-union, and secondary deformity should be corrected early with preservation of the joints whenever possible. Arthrodeses should be restricted to the affected joints. This article details the Hawkins classification, operative treatment and indications, and complications of fractures of the neck of the talus. It also discusses the treatment of fractures of the body of the talus and the talar head.

Häufigkeit von 0,3\% aller Brüche und von $3,4 \%$ aller Frakturen im Fußbereich angegeben. Sie entstehen, ähnlich wie die Brüche des Fersenbeins, durch Hochrasanztraumen wie Autounfälle oder Stürze aus großer Höhe.
Brüche des Sprungbeines sind aufgrund der anatomischen Gegebenheiten, insbesondere durch die Blutversorgung des Knochens, die am Sprungbein hauptsächlich über die Gelenkkapsel ohne eigene Gefäßversorgung erfolgt, schwierig zu behandeln und sollten nur von erfahrenen Operateuren versorgt werden. Die Gefäßversorgung kann bei Brüchen gestört werden, sodass es auch nach einer Operation, trotz primär gutem Ergebnis, zu einer Knochennekrose, d.h. zum Absterben des Knochens noch im Verlauf bis zu 3 Jahren kommen kann. Dies gilt auch aufgrund der häufigen Weichteilbeteiligung, die durch eine deutliche und schnelle Schwellung gekennzeichnet sein und somit eine Operation danach unmöglich machen kann.

Dieser Beitrag beschränkt sich auf die zentralen Talusfrakturen, d.h. die Frakturen des Talushalses und -körpers und die peripheren Frakturen des Taluskopfes. Die anderen peripheren Frakturen (Proc. lat., Proc. med., Proc. post.) werden nicht besprochen.

\section{Hauptteil}

\section{Topografische Anatomie}

Der Talus gehört zu den wenigen mobilen Knochen am Fuß. Zwischen 60 und $70 \%$ der Talusoberfläche sind mit Gelenkknorpel überzogen. Aufgrund dieser anatomischen Besonderheit ist die Blutversorgung des Talus problematisch.

Die arterielle Blutversorgung des Taluskörpers wird durch die Art. sinus tarsi, ein arterielles Gefäß vom Tarsalkanal versorgt. Dieses Gefäß wird von Ästen der Art. tibialis posterior und den Peronealarterien gebildet. Zusätzlich erfolgt die Versorgung durch Äste der A. deltiodea, die entlang des Lig. deltoideum ziehen. Die Arterien vom Tarsalkanal und Sinus bilden Anastomosen im Bereich des Talushalses. Äste der A. dorsa- 
lis pedis erreichen den Taluskopf und Talushals. Kleine Arterien in der Gelenkkapsel und den Bändern tragen zusätzlich etwas zur Blutversorgung des Talus bei. Der größte Anteil der Blutversorgung des Talus erfolgt durch die Arterien im Tarsalkanal. Sie versorgen direkt die Hälfte bis zu 2 Dritteln des Körpers und durch interne Anastomosen zum Teil auch den übrigen Bereich. Trotzdem erreicht der größte Teil der Blutversorgung den Untergrund des Halses und fließt nach posterior.

\section{Pathogenese}

Frakturen des Talushalses machen 50\% der Talusverletzungen aus. Historisch wurden sie auch als „aviator's astragalus" bekannt, da sie häufig im Zusammenhang mit Flugzeugabstürzen im Ersten Weltkrieg beobachtet wurden.

Typischerweise entstehen Talushalsfrakturen durch Hochrasanztraumen wie bei Pkw- oder Motorradunfällen oder bei Stürzen aus großer Höhe. Talushalsfrakturen entstehen bei einem Hochrasanztrauma und das tritt häufig bei jungen Erwachsenen auf. Männer sind mit 3 zu 1 häufiger betroffen als Frauen. Die 3. Lebensdekade wird überdurchschnittlich häufig betroffen. Zusätzlich werden Begleitverletzungen des muskuloskeletalen Systems (in 64\% der Hawkins-Frakturen) oder andere Organsysteme betroffen. Innenknöchelfrakturen werden bei $20-30 \%$ der berichteten Fälle, vor allem bei Frakturen mit herausgeschleudertem Taluskörper, gesehen. $10 \%$ erleiden zusätzlich eine Kalkaneusfraktur. Vielfach wird angenommen, dass die Fraktur durch eine übermäßige Dorsalflexion des Talushalses mit Anprall an die ventrale Tibiagelenkkante entsteht. Diese Fraktur im Labor nachzustellen, ist sehr schwierig. Die Fraktur durch forcierte Dorsalflexion an Leichenfüßen zu erstellen gelang bisher nicht. Peterson und Romanus gelang es, die Fraktur bei Neutralstellung im oberen Sprunggelenk unter Krafteinwirkung auf den Kalkaneus gegenüber dem darüberliegenden Talus und der Tibia zu generieren. Mit geringerer Kraft gelingt es, eine nicht dislozierte Talushalsfraktur zu erzeugen. Größere Kräfte produzieren eine Ruptur der posterioren talokalkanearen und interossären Bänder mit Subluxation des Subtalargelenkes. Der Kalkaneus wird nach anterior gedrückt, wobei er klassischerweise nach medial disloziert. Der Taluskörper geht in Richtung Equinus, und der Taluskopf wird dorsal des Halses disloziert. Bei fortgeführter Kraft werden die posterioren Bandverbindungen zur Sprunggelenksfraktur gedrückt und der Talus wird nach posteriomedial disloziert. Sneppen und Buhl beschreiben 26 Talushalsfrakturen bei einer Gruppe von 1806 Sprunggelenksfrakturen und fanden die Supinationskraft als die am häufigsten mit diesen kombinierten Verletzungen assoziierte Krafteinwirkung. Diese Ergebnisse werden auch von anderen Autoren geteilt.

\section{Diagnostik}

\section{Klinische Untersuchung}

Während der klinischen Untersuchung können Frakturdislokation und mögliche Luxationen durch die rasch einsetzende Schwellung maskiert werden. Dies macht die Palpation der Fraktur unmöglich. Bei Frakturen mit Extrusion des Taluskörpers wird die darüberliegende Haut häufig so überdehnt, dass sie schnell nekrotisch wird. Die neurovaskulären Strukturen werden normalerweise von schweren Verletzungen verschont. Trotzdem kann der Weichteilschaden erheblich sein und ein Kompartmentsyndrom am Unterschenkel oder Fuß kann sich entwickeln. Erste diagnostische Maßnahmen sind Inspektion (Schwellung), Palpation inkl. Prüfung der Gelenkstabilität, Prüfung der Durchblutung, Motorik und Sensibilität. Beim Verdacht auf ein Kompartmentsyndrom sollte eine Fußkompartmentdruckmessung durchgeführt werden (z.B. mit einem Intracompartmental Permanent Pressure Monitoring System, Stryker $^{\mathrm{TM}}$ Corporation, Santa Clara, CA, USA). Bei einer Druckdifferenz von weniger als $30 \mathrm{mmHg}$ zwischen dem diastolischen Blutdruck und dem Druck im Kompartment sollte eine Faszienspaltung erfolgen.

Der Ausschluss von Begleitfrakturen ist wichtig (Abb.1).

\section{Radiologische Untersuchung}

Das Vorliegen einer Talushalsfraktur wird normalerweise einfach im anterioposteriorem (a.-p.) und vor allem im lateralen Strahlengang erkannt. Die seitliche Ebene zeigt die Dislokation der Halsfraktur und die Inkongruenz von OSG, Subtalargelenk oder des Talonavikulargelenkes (Abb.1). Varus- oder Valgusfehlstellung des Talushalses sind in der normalen A.-p. Ebene oft schwierig

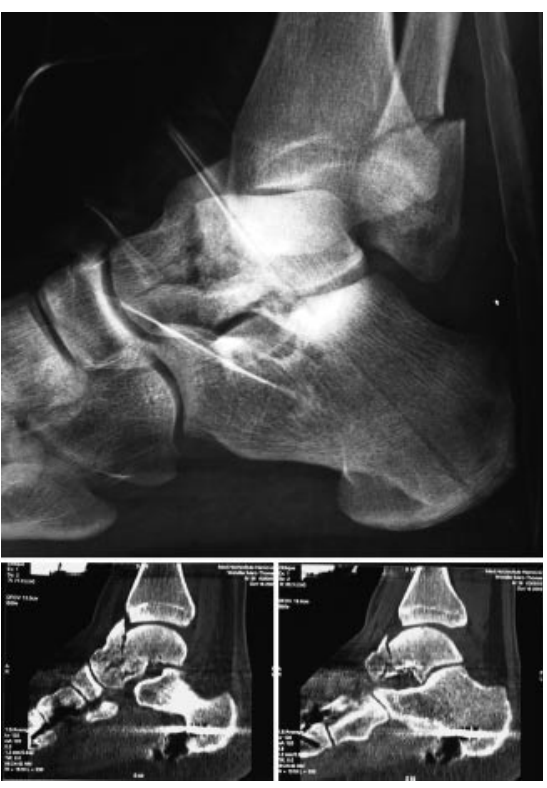

Abb. 1 Talushalsfraktur Typ Hawkins III mit inkompletter Taluskopffraktur bei einer OSGFraktur. Oben: laterale Aufnahme des dislozierten Talus mit OSG und Subtalargelenk. Unten: CT nach geschlossener Reposition.

zu sehen. Da Varusfehlstellungen häufig vorkommen, wurde von Canale und Kelly eine modifizierte A.-p. Röntgeneinstellung zur besseren Darstellung des Alignments des Talushalses in der Transversalebene beschrieben. Hierbei wird eine Röntgenkassette unter die Füße gelegt. Der Fuß ist im OSG maximal plantarflektiert und um 15 Grad proniert. Der Zentralstrahl trifft in 75 Grad auf die Kassette. Diese Einstellung ist sehr hilfreich für die Darstellung des Varus/Valgusalignements nach der Frakturreposition.

Bei vorliegender Talusfraktur ist eine Computertomografie zur genaueren Analyse der Fraktur dringend indiziert (Abb.1).

\section{Klassifikation}

Alle Klassifikationen unterscheiden die sog. zentralen Talusfrakturen, die Taluskopf, -hals oder -körper betreffen, von den sog. peripheren Frakturen: Diese betreffen den Proc. lateralis (,Snowboard ankle") und den Proc. posterior oder sind Abscher-Brüche an Talusdom oder-kopf.

Hawkins beschrieb 1970 eine Klassifikation von Talushalsfrakturen (Abb. 2). Sie basieren auf dem Ausmaß der Dislokation des Talushalses und der Kongruenz vom Subtalargelenk und oberen Sprunggelenk. 


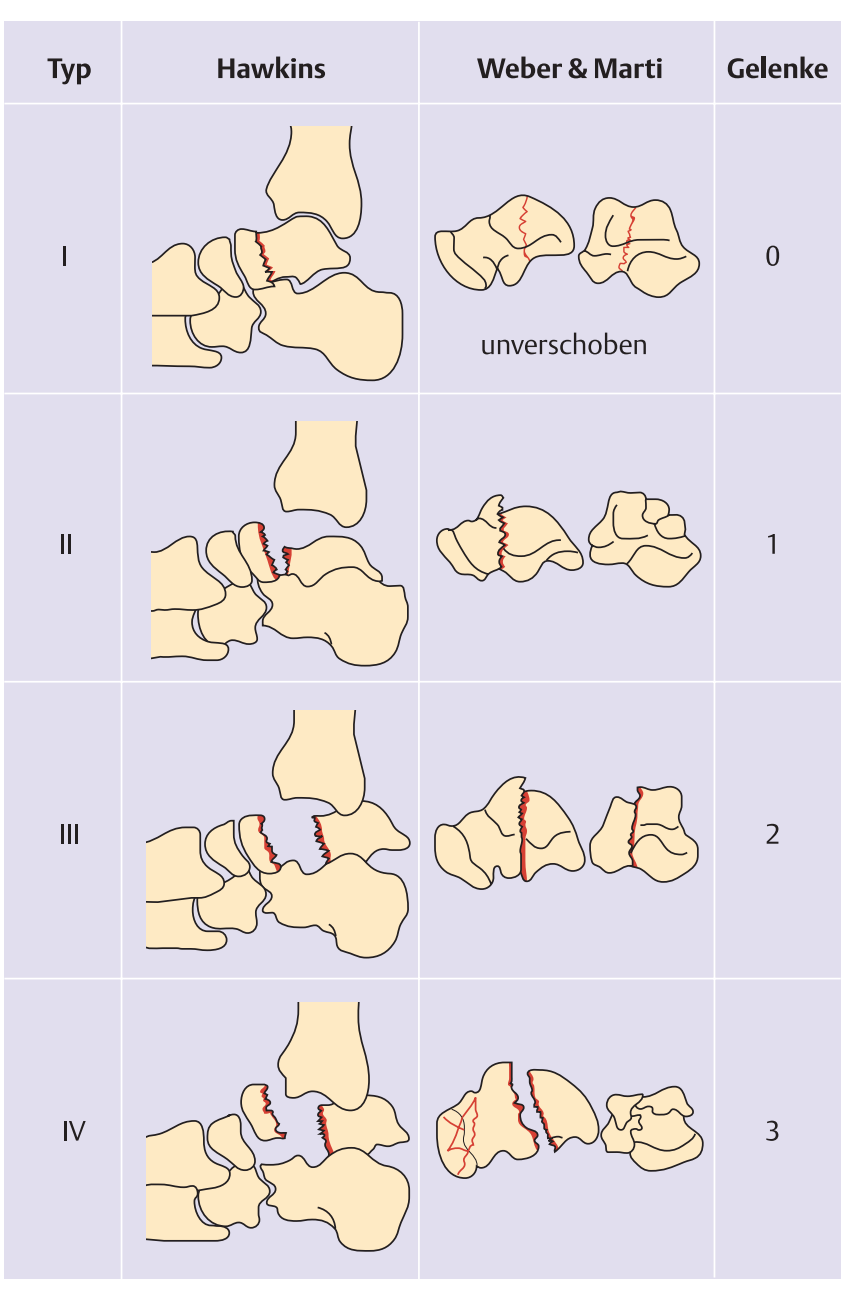

Abb. 2 Klassifikation nach Hawkins von Talushals- und -körperfrakturen unter Berücksichtigung der betroffenen Gelenke. die Kongruenz des OSG und Subtalargelenkes wiederherzustellen und das Risiko der avaskulären Nekrose durch Optimierung der verbleibenden Blutversorgung zu reduzieren. Diese Frakturen sind relativ selten, sodass sie von einzelnen Behandlern häufig nicht gesehen werden.

\section{Nicht operative Therapie}

Die nicht operative Behandlung ist angezeigt bei allen nicht dislozierten Frakturen. Diese Brüche sind entweder Gelenkfrakturen (Talusrolle, Taluskopf, Proc. lateralis) oder sie betreffen den Gleitweg vorbeiziehender Sehnen (Proc. posterior). Deshalb muss die tatsächliche Fragmentdislokation durch qualitativ einwandfreie CT-Aufnahmen exakt beurteilbar sein und sorgfältig kontrolliert werden. Zur nicht operativen Therapie dieser Brüche wird die verletzte Extremität zunächst durch eine Unterschenkelgipsschiene ruhig gestellt, die nach Abschwellung in einen zirkulären Unterschenkelliegegips umgewandelt wird. Nach 4 Wochen erfolgt der Wechsel auf einen Unterschenkelgehgips mit gut anmodellierter Sohle, nach insgesamt 8 Wochen die Freigabe des Beins.

Die Therapie der zentralen Frakturen ohne begleitende Gelenksluxationen hängt davon ab, ob diese Brüche mit einer Einstauchung einhergehen. In diesen Fällen ist eine operative Aufrichtung mit Spongiosaunterfütterung und Osteosynthese angezeigt. Alle anderen Fälle können nicht operativ behandelt werden. Die Anhänger einer äußeren Ruhigstellung auch aller Hawkins-I-Verletzungen empfehlen ebenfalls das für die peripheren Brüche angegebene Schema. Anhänger der funktionellen Behandlung erlauben nach initialer Ruhigstellung im Gipsverband für einige Tage den dann gipsfreien Sohlenkontakt für 8 Wochen. Anhänger der operativen Behandlung verschrauben auch Hawkins-I-Frakturen mit aufgeschobener Dringlichkeit und möglichst minimalinvasiv.

\section{Operative Behandlung}

Alle Frakturformen, die geschlossen nicht reponibel, nicht retinierbar oder mit Gelenkverwerfungen bzw. relevanter Verkürzung der lateralen/medialen Fußsäule einhergehen, sollten, sofern keine allgemeinen oder lokalen Kontraindikationen bestehen, mit offener Reposition und interner Fixation mit Schrauben und K-Drähten behandelt 


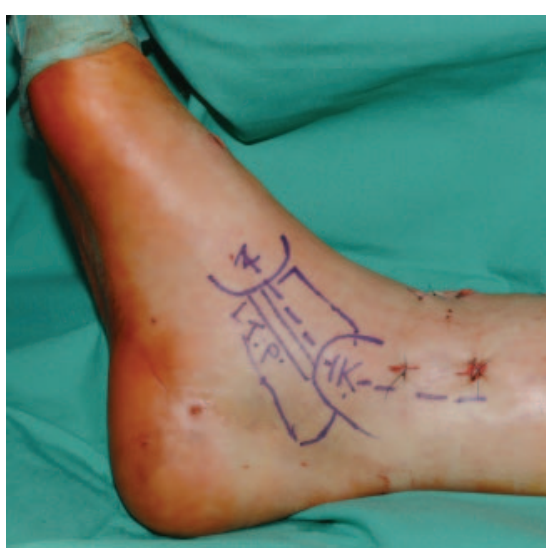

Abb. 3 Medialer Zugang zum Talushals. Gestrichelte Linie: Inzision; T.p.: M.-tibialis-posterior-Sehne; N: Os naviculare; IK: Malleolus medialis. Stichinzisionen nach Einbringen eines Tibianagels.

werden. Die operative Behandlung wird im Folgenden, um eine optimale Übersicht zu gewährleisten, in Form einer Checkliste dargestellt.

\section{Anästhesie}

- Regionalanästhesie (spinal/epidural/ popliteal) oder „Vollnarkose“

- Antibiotikaprophylaxe je nach häuslichem Standard (z.B. Cephalosporine 3. Generation).

Tisch und OP-Set

- 3,5-mm-Kortikalisschrauben und Spongiosaschrauben mit Stahl- oder Titaniumlegierung

- Standardosteosyntheseset, je nach internem Standard

- Röntgentisch

- Röntgengerät

- Karbontisch und dreidimensionales Röntgengerät wenn verfügbar (z.B. ARCADIS, Siemens AG, Erlangen).

Tisch Setting

- Instrumente stehen am Fußende des Tisches

- Röntgengerät sollte auf der kontralateralen Seite zur Verfügung stehen.

Patientenlagerung

- Rückenlage

- Blutsperre wird nicht empfohlen, da hohes Risiko einer ischämischen Wundnekrose.

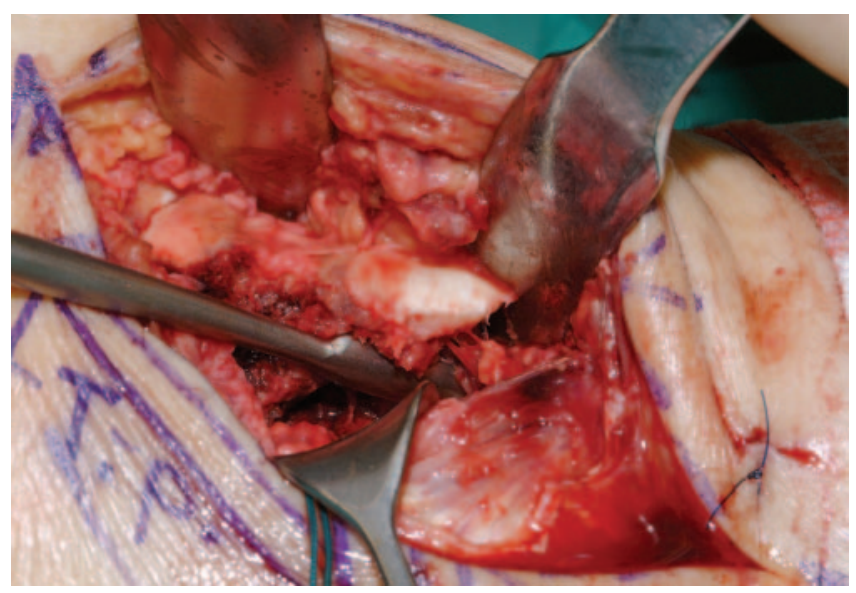

Abb. 4 Medialer Zugang bei Talushalsfraktur (Hawkins III). Fraktur wurde zur Spülung des Operationsfeldes mit einem Raspatorium verschoben.

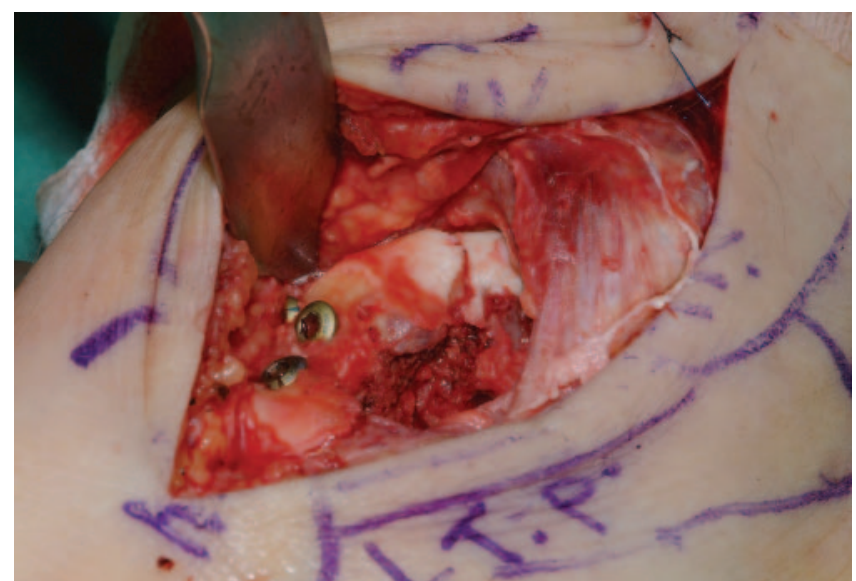

Abb. 5 Medialer Zugang bei Talushalsfraktur (Hawkins III). Fraktur nach offener Reposition und interner Fixation mit zwei 3,5-mm-Vollgewindeschrauben (Schrauben oben). Eine Schraube wurde zur Fixation der Taluskopffraktur eingebracht (Schraube unten).
Abdeckung

- Reinigung/steriles Abwaschen des Unterschenkels und des ganzen Fußes mit Desinfektionslösung, wobei eine gründliche Reinigung interdigital erfolgen sollte

- Steriles Abdecken ab der Mitte des Unterschenkels, zwischen Knie und Sprunggelenk

- Tape um den Vorfuß, um das Risiko einer Infektion zu minimieren.

\section{Operativer Zugang}

Bei den meisten Talusfrakturen ist der mediale Zugang Standard (Abb. 3 bis 5). Ein kombinierter medialer und lateraler Zugang sollte gewählt werden, wenn die Reposition und interne Fixation über den medialen Zuganges nicht möglich ist.

- Der mediale Zugang (Abb.3 bis 5) sollte vom anterioren Teil des medialen Innenknöchels bis zum dorsalen Teil der Tuberositas ossis navicularis verlaufen (Abb.3). Der Hautschnitt ist in die Tiefe bis zum Knochen zu führen, genau bis zur Sehne des M. tibialis posterior. Eine Durchtrennung des Lig. deltoideum sollte nicht erfolgen, da es den Ramus deltoideus der A. tibialis posterior zur Versorgung des mittleren Taluskörpers mit sich führen kann.

- Für die Versorgung von Taluskörperfrakturen kann eine Innenknöchelosteotomie nötig sein.

- Innerhalb der Inzision sollte die Fraktur dargestellt, eine Hämatomausräumung erfolgen und die Fraktur mobilisiert werden (Abb. 4 und $\mathbf{5}$ ).

- Schonung der dorsalen oder plantaren Weichteile vom Talushals

- Der laterale Zugang (Abb.6) liegt an der vorderen Begrenzung der Fibulaspitze und verläuft in den Hautspaltlinien. Im zentralen Zugangsbereich verläuft das Lig. fibulotalare ant. und an der dorsalen Begrenzung des $\mathrm{Zu}$ gangs der N. suralis.

- Innerhalb der Inzision(-en) sollte die Fraktur dargestellt, eine Hämatomausräumung erfolgen und die Fraktur mobilisiert werden (Abb. 4 und $\mathbf{6}$ ).

- Schonung der dorsalen oder plantaren Weichteile. 


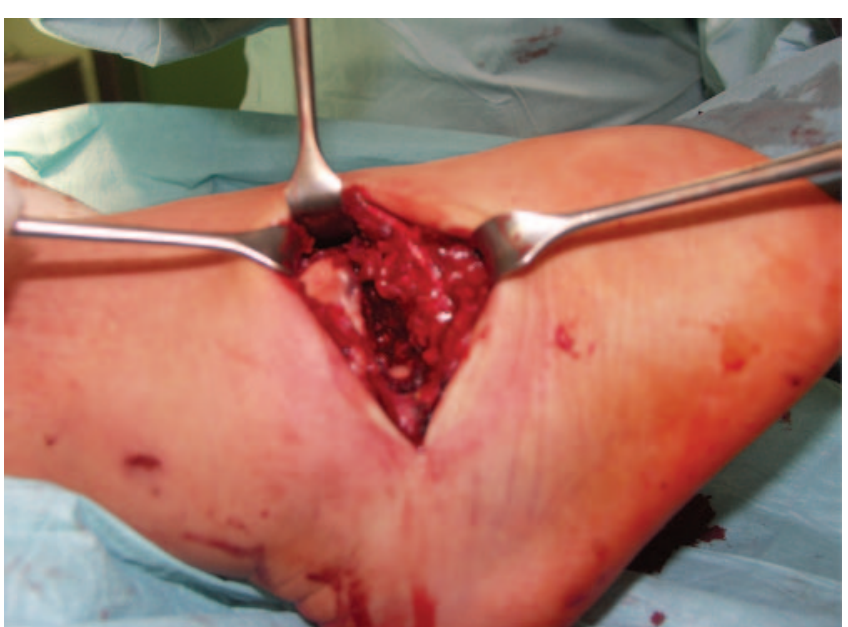

Abb. 6 Lateraler Zugang mit Talushalsfraktur; anderer Fall wie Abb. 3-5 und 7, selber Fall wie Abb. 8.

Reposition und interne Fixation

Die Fraktur sollte unter Sichtkontrolle reponiert werden.

- Ausschluss von Frakturfragmenten am medialen Talushals, da dadurch am lateralen Talushals eine Varusfehlstellung und damit eine Höhendifferenz oder Lücke auf der Frakturseite verbleiben kann. Um eine adäquate Wiederherstellung zu erreichen sollte deshalb, nach provisorischer Reposition, eine temporäre Stabilisierung mit 2,0-mm-K-Drähten erfolgen.

- Nach der provisorischen Drahtstabilisierung sollte eine Varus- oder Supinationsfehlstellung des Fußes ausgeschlossen werden.

- Intraoperative Kontrolle des Repositionsergebnisses durch laterale, anteroposteriore und kanale Aufnahmen.

- Nach adäquater Reposition sollte mit Schrauben eine definitive Fixation erfolgen.

- Mehrfachfragmente sind an der medialen Seite häufig. Jedoch sollte hier keine Fixation durch eine Zugschraube erfolgen, da dadurch eine Varusstellung entstehen könnte. Eine harte Kortikalis entlang des dorsal gelegenen Sinus tarsi erlaubt eine sehr gute Fixation von Schrauben, sodass mindestens 2 Schrauben vom medialen Talushals quer zur Frakturseite eingebracht werden können (Abb. 6 bis $\mathbf{8}$ ).

- Titaniumschrauben lassen postoperativ ein MRT zum Ausschluss einer avaskulären Nekrose zu. Es können auch Stahlschrauben eingesetzt werden, jedoch muss hier bei einem MRT mit Signalstörungen in der Nähe der Schrauben gerechnet werden, die die Darstellung von Teilen des Talus verhindern könnten.

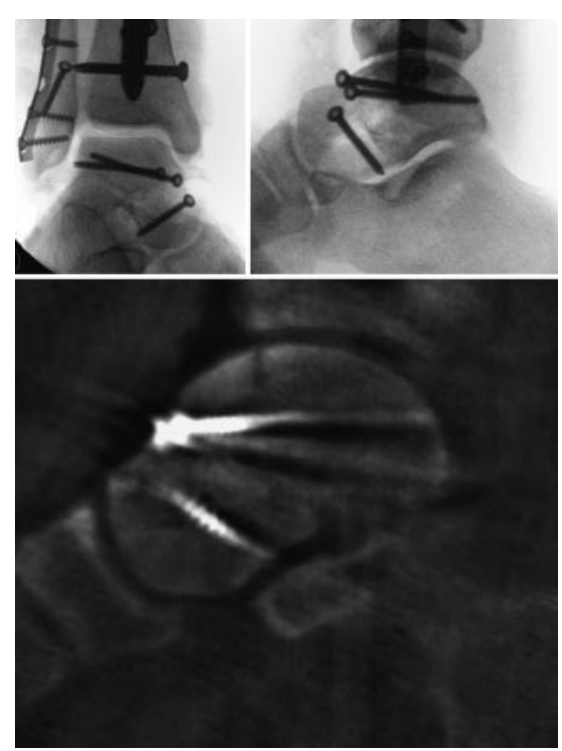

Abb. 7 Talushalsfraktur (Hawkins III) und Taluskopffraktur. Fraktur nach offener Reposition und interner Fixation mit zwei 3,5-mmVollgewindeschrauben (Schrauben oben). Eine Schraube wurde zur Fixation der Taluskopffraktur eingebracht (Schraube unten). Versorgung einer Begleitfraktur der Tibia mit einem Tibianagel und der Fibula mit Plattenosteosynthese. Unten Darstellung mit einem Röntgengerät für dreidimensionale Aufnahmen (ISO-C-3D bzw. ARCADIS, Siemens AG, Erlangen).

- Liegt eine Fraktur am distalen Talushals vor, so sollten die Schrauben im Taluskopf versenkt werden. Als Alternative können zur Fixation Polylactic Acid (PLA), bioabsorbierbare Schrauben (Bionix Implants, Inc., Blue Beil, Pennsylvania, USA), Herbert-Schrauben (Zimmer Corp., Warsaw, Illinois, USA), oder Acutrak-Schrauben (Acumed, Beaverton, Oregon, USA) in anteroposteriorer Richtung eingebracht werden. Obwohl bei einem postero-
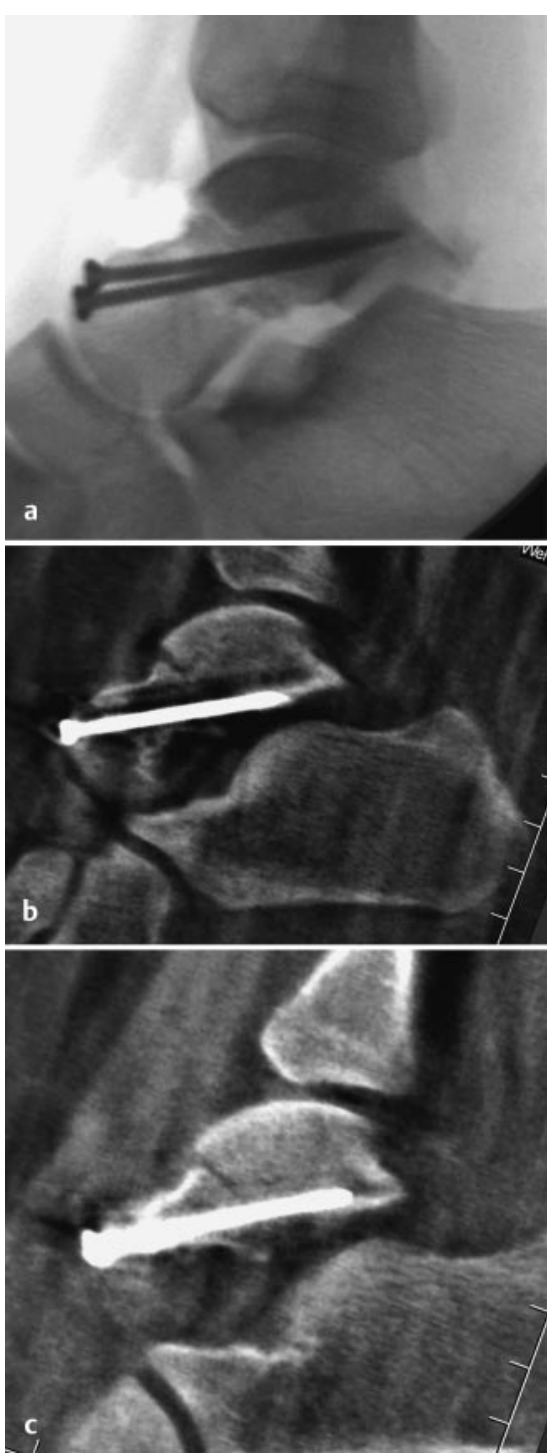

Abb. 8a bis c Korrektur einer intraartikulären Schraube im Subtalargelenk. Intraoperative Aufnahmen mit einem Röntgengerät für dreidimensionale Aufnahmen (ISO-C-3D bzw. ARCADIS, Siemens AG, Erlangen). a Bild des Röntgenbildverstärkers. b Parasagittale Reformation aus ARCADIS-Scan desselben Zustands wie in a mit jetzt erkennbar intraartikulär gelegener Schraubenspitze. c Zweiter ARCADIS-Scan nach Korrektur der Schraubenlage mit nicht mehr intraartikulärer Lage.

lateralen Zugang die Platzierung der Schrauben von der Tuberositas posterior zum Taluskopf zu einer hohen Stabilität der Fraktur führt, ist die Reposition bei diesem Zugang eine Herausforderung.

- Die intraoperative Bildgebung erfolgt in den oben beschriebenen Standardebenen und wenn möglich, mit einem Röntgengerät für dreidimensionale Aufnahmen (z.B. ARCADIS, Siemens AG, Erlangen, Abb. 7 und 8). 
Wundverschluss

- Gründliche Spülung und Blutstillung

- Einlage einer Redon-Drainage (12 F) und Adaptation der subkutanen Faszie (2.0 PDS/Vicryl)

- Hautverschluss mit Stapler oder monofilem, nicht absorbierbarem Nahtmaterial

- Anlage eines Unterschenkelspaltgipses.

\section{Postoperative Versorgung}

- Entfernung der Redon-Drainage nach 48 Stunden

- Frühfunktionelle Teilbelastung mit $15 \mathrm{~kg}$ und Physiotherapie.

Kontrolluntersuchungen

- Wiedervorstellung nach 6 und 12 Wochen mit Röntgenkontrolle

- Nach Röntgenkontrolle nach 6 Wochen Übergang zur Vollbelastung

- Nach klinischer und radiologischer Dokumentation eines guten Heilungsprozesses muss eine weitere Nachuntersuchung nur auf Bitte um Wiedervorstellung durch einen niedergelassenen Chirurgen erfolgen

- Wiedervorstellung mit Röntgenbildern nach 1 Jahr zum Ausschluss einer Taluskopfnekrose.

Nachbehandlung

Wenn immer möglich, sollte diese frühfunktionell mit passiver OSG-Bewegungsschiene und unter aktiver Krankengymnastik mit Teilbelastung des Fußes von $15 \mathrm{~kg}$ erfolgen.

Die Vollbelastung ist je nach Frakturtyp in der Regel nach 2-6 Wochen möglich.

Bei Trümmerfrakturen ist je nach Rekonstruierbarkeit die frühsekundäre USG- oder OSG/USG-Arthrodese anzustreben. Bei gut rekonstruiertem Talus und großer Wahrscheinlichkeit einer Talusnekrose (Typ IV) kann ein Allgöwer-Apparat für 6-2 Monate empfohlen werden, um eine Revaskularisierung ohne sekundäre Deformation zu ermöglichen. Ein Beweis für die Notwendigkeit einer Entlastung über die 12. Woche hinaus besteht jedoch nicht.
Komplikationen

Die häufigste und in der Regel schicksalhafte Komplikation stellt die partielle oder totale Talusnekrose dar, die nach der Literatur entsprechend der erweiterten Hawkins-Klassifikation und der Marti-und-Weber-Einteilung beim Typ I $0-3 \%$, beim Typ II $0-20 \%$, beim Typ III 20-100\% und beim Typ IV $50-100 \%$ beträgt.

Bei Hawkins-III- und -IV-Frakturen wird die Blutzufuhr des Talus mit der Ausnahme der deltoidalen Äste, die durch die Dislokation oder Rotation des Talus abknicken können, komplett zerstört.

\section{Prognose}

Die höheren Grade der Klassifikation folgen einem größeren Trauma und einer schlechteren Langzeitprognose.

Die Prognose einer zentralen Talusfraktur ist direkt abhängig vom Frakturtyp, d.h. vom Luxationsgrad, dem Ausmaß der direkten Taluszerstörung, der Gelenkbeteiligung, vom primär begleitenden Weichteilschaden und vom sekundären chirurgenbezogenen Trauma (frustranes geschlossenes oder insuffizientes offenes Vorgehen). Hawkins-IFrakturen haben die beste Prognose und heilen normalerweise ohne Verzögerung. Nach Zwipps Analyse sind vor allem Frühkomplikationen im Sinne der aseptischen/septischen Talusnekrose von den vorgenannten Kriterien abhängig, posttraumatische Arthrosen, vor allem jedoch vom Frakturtyp allein. So ist beim Typ IV mit einer 3-Gelenk-Beteiligung trotz guter Rekonstruktion in jedem 7. Fall mit einer konsekutiven Arthrose des OSG/USG zu rechnen.

Für den postoperativen Verlauf hinsichtlich einer Talusnekrose erscheint das sog. Hawkins-Zeichen von großer Bedeutung, da es bei subchondraler Demineralisation nach ca. 3 Wochen eine verbliebene Durchblutung des Talus anzeigt. Die Knochenszintigrafie hat sich zu dieser Fragestellung nicht bewährt, das MR ist bei liegenden Implantaten in der Regel nicht beurteilbar, weswegen in jüngster Zeit Titanschrauben verwendet werden. Zur Vitalitätsanalyse des Talus ist die PET-CT (Positronen-Emissionstomografie kombiniert mit Computertomografie) am besten geeignet.

\section{Schlussfolgerung}

Talusfrakturen gehören zu den seltenen Fußfrakturen, führen aber potenziell zu schweren Komplikationen. Alle dislozierten Talusfrakturen stellen eine absolute Operationsindikation dar. Die anatomische Reposition der Gelenkflächen und der Talusform und die Stabilisierung mit Zugschrauben stellt meistens die Methode der Wahl dar.

\section{Literatur}

1 Adelaar RS. The treatment of complex fractures of the talus. Orthop Clin North Am 1989; 20: $691-707$

2 Attiah M, Sanders DW, Valdivia G, Cooper I, Ferreira L, MacLeod MD, Johnson JA. Comminuted talar neck fractures: a mechanical comparison of fixation techniques. J Orthop Trauma 2007; 21: 47-51

${ }^{3}$ Berlet GC, Lee TH, Massa EG. Talar neck fractures. Orthop Clin North Am 2001; 32: 53-64

${ }^{4}$ Canale ST, Kelly FB Jr. Fractures of the neck of the talus. Long-term evaluation of seventyone cases. J Bone Joint Surg [Am] 1978; 60: $143-156$

5 Ebraheim NA, Mekhail AO, Salpietro BJ, Mermer MJ, Jackson WT. Talar neck fractures: anatomic considerations for posterior screw application. Foot Ankle Int 1996; 17: $541-547$

${ }^{6}$ Hawkins LG. Fractures of the neck of the talus. J Bone Joint Surg [Am] 1970; 52: 991 1002

7 Pennal GF. Fractures of the talus. Clin Orthop 1963; 30: 53-63

8 Rammelt S, Winkler J, Grass R, Zwipp H. Reconstruction after talar fractures. Foot Ankle Clin 2006; 11: $61-84$

${ }^{9}$ Rammelt S, Winkler J, Heineck J, Zwipp H. Anatomical reconstruction of malunited talus fractures: a prospective study of 10 patients followed for 4 years. Acta Orthop 2005; 76: 588 - 596

10 Thordarson DB. Talar body fractures. Orthop Clin North Am 2001; 32: 65 - 77

\section{Prof. Dr. med. Martinus Richter Chefarzt \\ Stefan Zech}

Facharzt

II. Chirurgische Klinik (Unfallchirurgie, Orthopädie und Fußchirurgie)

Klinikum Coburg

Ketschendorfer Straße 33

96450 Coburg 